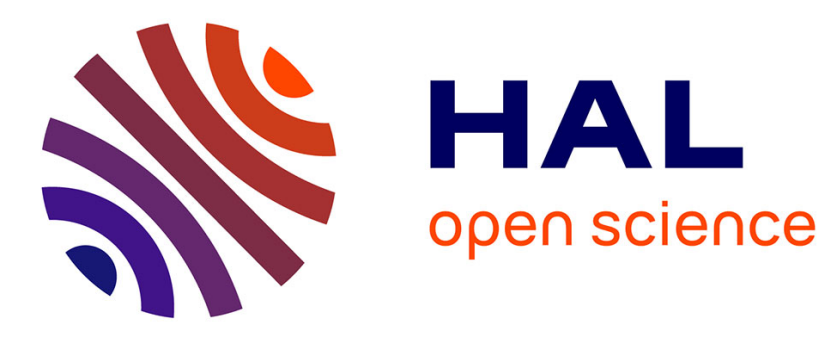

\title{
Review of Double Beta Decay Experiments
}

Laurent Simard

\section{To cite this version:}

Laurent Simard. Review of Double Beta Decay Experiments. 17th Lomonosov Conference on Elementary Particle Physics, Aug 2015, Moscow, Russia. pp.140-146, 10.1142/9789813224568_0021. hal-01555192

\section{HAL Id: hal-01555192 \\ https://hal.science/hal-01555192}

Submitted on 26 Jun 2020

HAL is a multi-disciplinary open access archive for the deposit and dissemination of scientific research documents, whether they are published or not. The documents may come from teaching and research institutions in France or abroad, or from public or private research centers.
L'archive ouverte pluridisciplinaire HAL, est destinée au dépôt et à la diffusion de documents scientifiques de niveau recherche, publiés ou non, émanant des établissements d'enseignement et de recherche français ou étrangers, des laboratoires publics ou privés. 


\title{
REVIEW OF DOUBLE BETA DECAY EXPERIMENTS
}

\author{
Laurent Simard ${ }^{a}$ \\ LAL, Univ Paris-Sud, CNRS/IN2P3, Université Paris-Saclay, Orsay, France \\ Institut Universitaire de France, F-75005 Paris, France
}

\begin{abstract}
The search of the neutrinoless double beta decay is currently the only way to test the nature, Dirac or Majorana, of the neutrino. Two different experimental approaches exist : one, where source and detector coïncide, is pure calorimetric. In the other technique, before the measurements of their energies, the two electrons are tracked. The results of the currently data-taking experiments are presented. The expected sensitivities for future experiments are discussed.
\end{abstract}

\section{Majorana neutrinos and double beta decay}

As a neutral fermion, it can be identical to its antiparticle (= Majorana particle). The Majorana mass term of neutrino is naturally added in a simple extension of the standard model. It could explain the baryon-antibaryon asymmetry by leptogenesis. The most sensitive process to search for the Majorana nature of the neutrinos is the neutrinoless double beta decay $(0 \nu 2 \beta)$, a lepton number violating process $(\Delta \mathrm{L}=2):(\mathrm{A}, \mathrm{Z})->(\mathrm{A}, \mathrm{Z}+2)+2 \mathrm{e}^{-}$
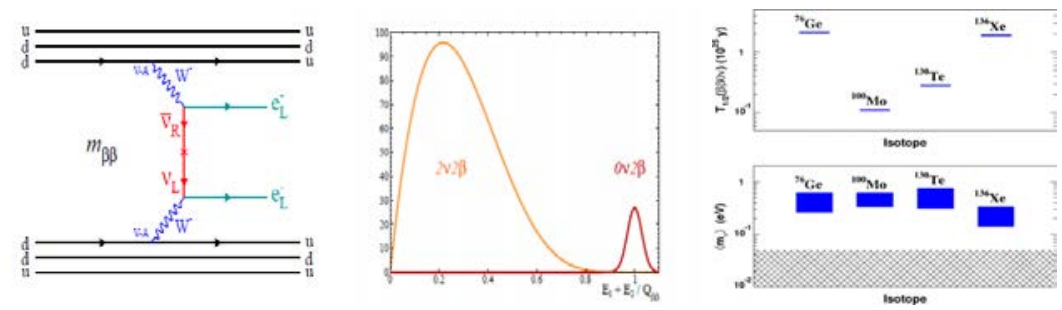

Figure 1: Top : $0 \nu 2 \beta$ mechanism of exchange of a light Majorana neutrino . Middle : 2electron energy spectra for $\beta \beta$ processes. Right : sensitivities of $\beta \beta$ decay experiments to various isotopes.

For the exchange of a light Majorana neutrino (see left plot of Figure 1) the half-life $\mathrm{T}_{1 / 2}^{0 \nu}$ of the process is related to the effective neutrino Majorana mass $\mathrm{m}_{\beta \beta}: \frac{1}{\mathrm{~T}_{1 / 2}^{0 \nu}}=\mathrm{G}_{0 \nu}\left|\mathrm{M}_{0 \nu}\right|^{2}\left|\mathrm{~m}_{\beta \beta}\right|^{2}$, where $\mathrm{G}_{0 \nu}$ is the phase space factor and $\mathrm{M}_{0 \nu}$ the nuclear matrix element. The process has not been observed so far, and limits on the half-life are tuypically $10^{24}-10^{25} \mathrm{y}$. Several mechanisms can generate the $0 \nu 2 \beta$ process : light Majorana neutrino exchange $\mathrm{m}_{\beta \beta}$, right handed current, Majoron and SUSY. Experimentally, a peak in the 2-electron energy spectrum is expected at $\mathrm{Q}_{\beta \beta}$. The current best limits on the $0 \nu \beta \beta$ search are given in the right plot of Figure 1.

${ }^{a}$ E-mail: simard@lal.in2p3.fr 


\section{Recent results or progress on the double beta search}

\subsection{Germanium detectors}

\section{GERDA}

The principle is to use bare Ge diodes in liquid argon enriched in ${ }^{76} \mathrm{Ge}$ at $86 \%$. A gradual deployment of the detector strings in the cryostat is performed in Gran Sasso (3800 mwe). The background target is 0.01 count/(keV.kg.y).

The Phase 1 data taking (2011-2013) used $18 \mathrm{~kg}$ of ${ }^{76} \mathrm{Ge}(8$ old semi-coaxials (HdM-IGEX) and 5 new BEGe.) The energy resolution FWHM at $\mathrm{Q}_{\beta \beta}$ obtained was [4.2-5.7] $\mathrm{keV}$ for coaxials and [2.6-4.0] keV for BEGe. With a $21.6 \mathrm{~kg} . \mathrm{y}$ exposure, using pulse shape discrimination, the limits sets were : $\mathrm{T}_{1 / 2}^{0 \nu}>2.110^{25} \mathrm{y}(90 \% \mathrm{CL})$ and $\left|\mathrm{m}_{\beta \beta}\right|<[0.2-0.4] \mathrm{eV}[1]$.
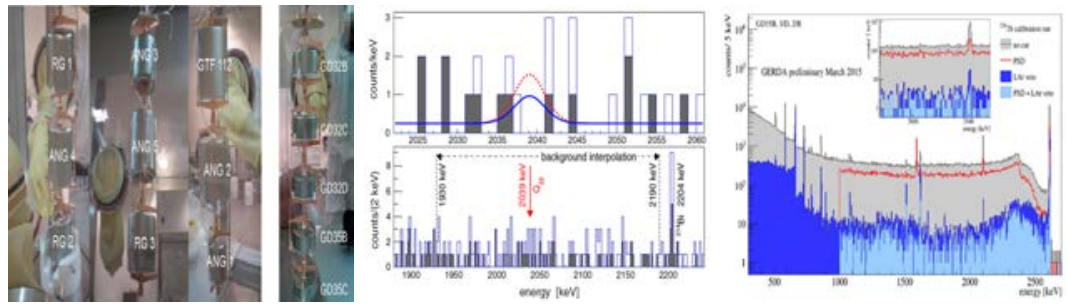

Figure 2: GERDA. Left : Detectors used for phase 1. Middle : Energy spectrum of Phase 1. Right : Energy spectrum with a ${ }^{228}$ Th source, for Phase2 detectors.

For the phase 2, $50 \mathrm{~kg}$ of ${ }^{76} \mathrm{Ge}$ will be used. The expected level of background is $10^{-3}$ count $/(\mathrm{keV} \cdot \mathrm{kg} . \mathrm{y})$. With a $100 \mathrm{~kg} . \mathrm{y}$ exposure the expected sensitivity is $\mathrm{T}_{1 / 2}^{0 \nu}>10^{26} \mathrm{y}$ for 30 new BEGe diodes due to several improvments : better PSD than for coaxials, use of PSD single site $(0 \nu 2 \beta) /$ multisites ( $\gamma$-rays) or surface $\left({ }^{42} \mathrm{~K}\right), \mathrm{FWHM} \simeq 3 \mathrm{keV}$ at $\mathrm{Q}_{\beta \beta}$ and detection of liquid argon scintillation light to veto backgrounds.

A possible extrapolation to higher masses will be estimated. The commissioning has started (spectrum from a ${ }^{228} \mathrm{Th}$ source in Figure 2.1.)

\section{Majorana}

The MAJORANA detector consists in enriched HpGe detectors in electroformed copper in Sanford underground lab. The target background level for 1 ton of ${ }^{76} \mathrm{Ge}$ detectors is 1 count/(ROI.kg.yr) for a $4 \mathrm{keV}$ ROI. A $40 \mathrm{~kg}$ demonstrator is under construction to demonstrate the ability to reach the necessary background level. Early 2015 half of enriched HpGe detectors and some natural detectors $(20 \mathrm{~kg}$ ) are completed. Late 2015 the rest of the HpGe detectors will be implemented. 

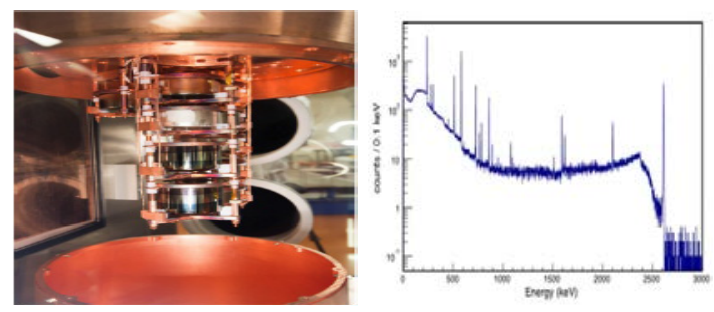

Figure 3: MAJORANA. Middle : One detector. Right : Spectrum obtained with a ${ }^{228} \mathrm{Th}$ source.

\subsection{Bolometers}

\section{Cuore}

The CUORE detector is made of $206 \mathrm{~kg}$ of ${ }^{130} \mathrm{Te}$ bolometers. The assembly and cleaning techniques have been improved, as well as the cryostat radiopurity. The target background level is $10^{-2}$ counts/(keV.kg.y), with a FWHM of $5 \mathrm{keV}$ at $\mathrm{Q}_{\beta \beta}$. The expected sensitivity is $\mathrm{T}_{1 / 2}^{0 \nu}>10^{26} \mathrm{y}$ in 5 years of data taking.

The first tower of CUORE holds $11 \mathrm{~kg}$ of ${ }^{130} \mathrm{Te}$ in the same cryostat as CUORICINO, with the same $\gamma$ background. The improved crystals supports induces a $\alpha$ background reduction. With a $9.8 \mathrm{~kg} . \mathrm{y}$ exposure the combined results with CUORICINO are : $\mathrm{T}_{1 / 2}^{0 \nu}>410^{24} \mathrm{y}(90 \% \mathrm{CL})$ and $\left|\mathrm{m}_{\beta \beta}\right|<[0.27-0.76] \mathrm{eV}$.
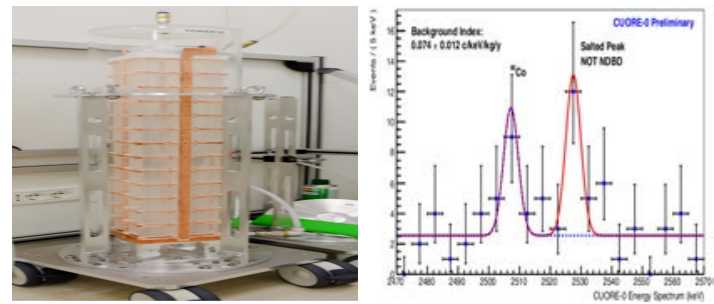

Figure 4: CUORE. Left : One tower. Middle : Spectrum of the CUORE-0 demonstrator.

\section{Lucifer}

The Lucifer project consists in 50 ZnSe crystals (18 kg of $\left.{ }^{82} \mathrm{Se}\right)$. The CUORICINO cryostat will be used after the end of CUORE-0 data taking The expected sensitivity is $\mathrm{T}_{1 / 2}^{0 \nu}>10^{26} \mathrm{y}$ in 5 years of data taking. The background measurement is ongoing with natural crystals in Gran Sasso. Some difficulties concern the reproducibility during the production of $\mathrm{ZnSe}$ crystals. 

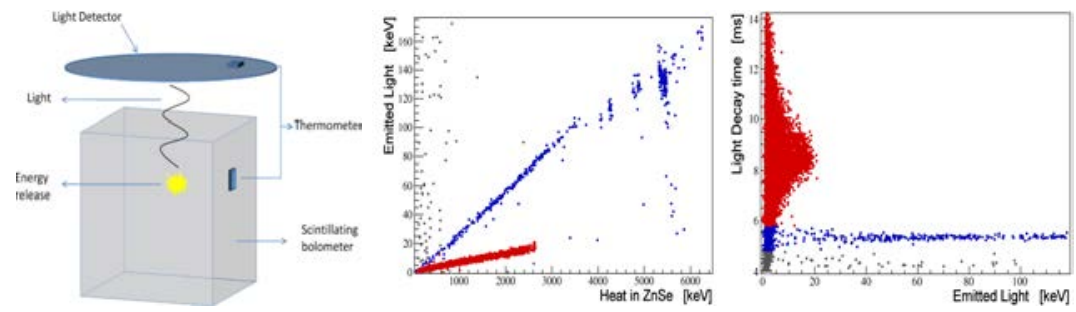

Figure 5: LUCIFER. Left : Principle of scintillating bolometers. Middle : Light versus heat signals. Right : Light decay time vs emitted light.

\section{Lumineu}
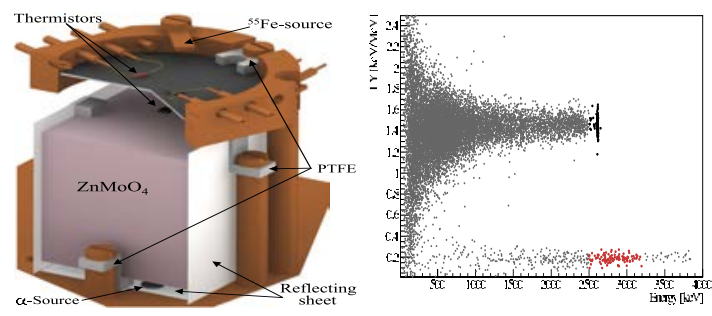

Figure 6: LUMINEU. Left : $\mathrm{Zn}^{100} \mathrm{MoO}_{4}$ scintillating bolometer. Right : Light vs heat signals.

The Goal is to build a demonstrator with 40 crystals ( $\simeq 7 \mathrm{~kg}$ of ${ }^{100} \mathrm{Mo}$ ). The expected sensitivity is $\mathrm{T}_{1 / 2}^{0 \nu}>1.210^{25} \mathrm{y}$ in 2 years. 20 crystals will be placed at Gran Sasso in early 2016 and 30 crystals in LSM in end 2016. A clear $\alpha$ and $\beta / \gamma$ discrimination is observed at LSM for the first natural and enriched crystals. The radiopurities of the natural and enriched crystals are very good.

\section{$2.3 T P C: E X O-200 / n E X O$}

EXO-200 benefits from the easy and cheap ${ }^{136}$ Xe enrichment (80\%). It hosts $200 \mathrm{~kg}$ of liquid xenon in a TPC at WIPP. With a fiducial volume of $79.4 \mathrm{~kg}$ of ${ }^{136} \mathrm{Xe}$, a background level of $(1.7 \pm 0.2) 10^{-3}$ counts/(keV.kg.y), a FWHM of $89 \mathrm{keV}$ at $\mathrm{Q}_{\beta \beta}$, the observed limit is : $\mathrm{T}_{1 / 2}^{0 \nu}>1.110^{25} \mathrm{y}(90 \% \mathrm{CL})$ and $\left|\mathrm{m}_{\beta \beta}\right|<$ $[0.19-0.45] \mathrm{eV}$ with an exposure of $123.7 \mathrm{~kg} . \mathrm{y}$ [3].

The nEXO project will be a liquid Xenon TPC in SNOLAB Cryopit. It will host $5 \mathrm{t}$ enriched at $90 \%$ in ${ }^{136} \mathrm{Xe}$. The baseline FWHM $86 \mathrm{keV}$ at $\mathrm{Q}_{\beta \beta}$ Scaling EXO-200 background with an improved discrimination, the expected sensitivty is $\mathrm{T}_{1 / 2}^{0 \nu}\left({ }^{136} \mathrm{Xe}\right)>4.110^{27} \mathrm{y}\left(2.110^{28} \mathrm{y}\right.$ with the tagging of the $\mathrm{Ba}$ ion $)$ in 10 years of data taking. 

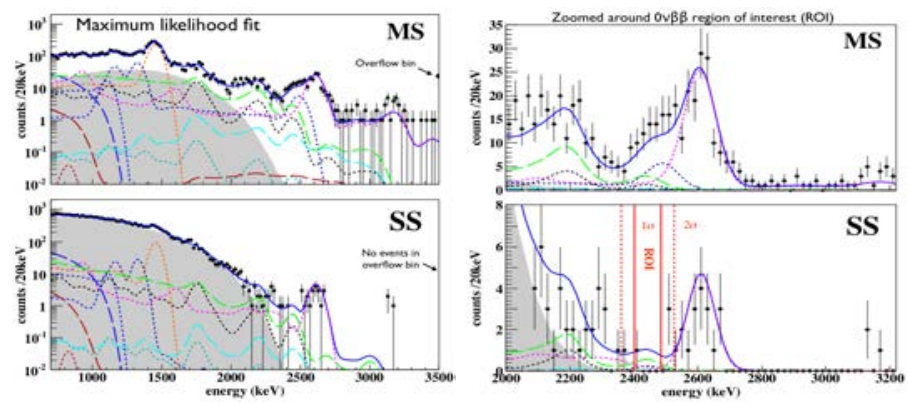

Figure 7: EXO-200. Left : Energy spectrum for single site(top) and multisite (bottom) events. Right : Same plots around $\mathrm{Q}_{\beta \beta}$.

\subsection{Scintillators :}

\section{KamLand-Zen}

13 tons of Xe-loaded liquid scintillator are hold in KamLand mini balloon (320 $\mathrm{kg}$ of enriched $\mathrm{Xe}$ ). The energy resolution is $232 \mathrm{keV}$ at $\mathrm{Q}_{\beta \beta}$. The first phase (2011-2012) has shown a dominant background from ${ }^{110 m} \mathrm{Ag}$. After purification of the liquid scintillator, the second phase (2013-2014) has demonstrated a more than 10 times reduction of ${ }^{110 m} \mathrm{Ag}$ background. The combined limit is : $\mathrm{T}_{1 / 2}^{0 \nu}>2.610^{25} \mathrm{y}(90 \% \mathrm{CL})$ and $\left|\mathrm{m}_{\beta \beta}\right|<[0.14-0.28] \mathrm{eV}$ with a $198.3 \mathrm{~kg} . \mathrm{y}$ exposure [4]. In the future, a miniballoon will be rebuilt, the Xe mass increased to $600 \mathrm{~kg}$ and the balloon radioactivity reduced.

\section{$\mathrm{SNO}+$}

The SNO detector will be filled with $800 \mathrm{~kg}$ of ${ }^{130} \mathrm{Te}(160 \mathrm{~kg}$ within the fiducial volume). The liquid scintillator loaded with Te has a high light yield : FWHM is $265 \mathrm{keV}$ at $\mathrm{Q}_{\beta \beta}$. The $\mathrm{T}_{1 / 2}^{2 \nu}\left({ }^{130} \mathrm{Te}\right)$ is high, inducing a low $2 \nu 2 \beta$ background. The liquid scintillator must be ultra radiopure in ${ }^{238} \mathrm{U}$ and ${ }^{232} \mathrm{Th}$. The neutrinos from solar ${ }^{8} \mathrm{~B}$ are the ultimate background. The expected sensitivity is $\mathrm{T}_{1 / 2}^{0 \nu}>9.410^{25} \mathrm{y}(90 \% \mathrm{CL})$.

\subsection{Tracko-calo : NEMO-3/SuperNEMO}

\section{NEMO-3}

The NEMO-3 detector was located in the Laboratoire Souterrain de Modane in France under 4800 m.w.e. shielded by $30 \mathrm{~cm}$ of borated water and wood, 19 $\mathrm{cm}$ of pure iron and radon-free air tent (after 2004). The $0 \nu 2 \beta$ events are fully reconstructed (individual electron energies, electron angular distribution) and 

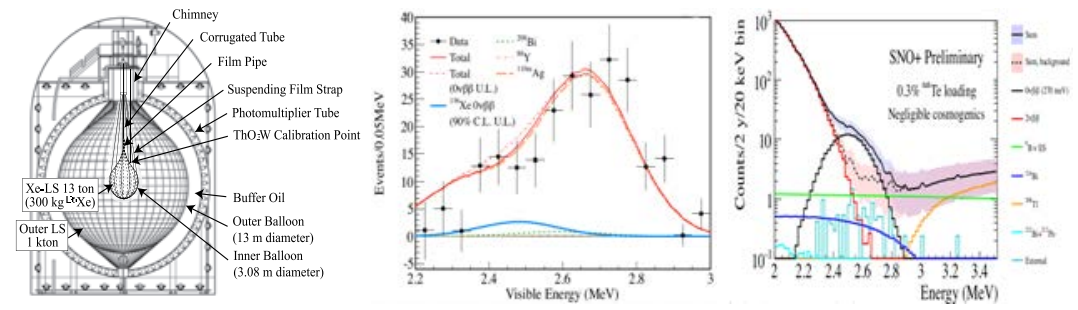

Figure 8: Left : Scheme of KamLand-Zen detector. Middle : KamLand-Zen spectrum around $\mathrm{Q}_{\beta \beta}$. Right : Expected $\mathrm{SNO}+$ spectrum around $\mathrm{Q}_{\beta \beta}$.

the background powerfully rejected. For ${ }^{100} \mathrm{Mo}$ with an exposure $34.7 \mathrm{~kg} . \mathrm{y}$, the limits are [5]: $\mathrm{T}_{1 / 2}^{0 \nu}>1.110^{24} \mathrm{y}(90 \% \mathrm{CL})$ and $\left|\mathrm{m}_{\beta \beta}\right|<[0.33-0.62] \mathrm{eV}$
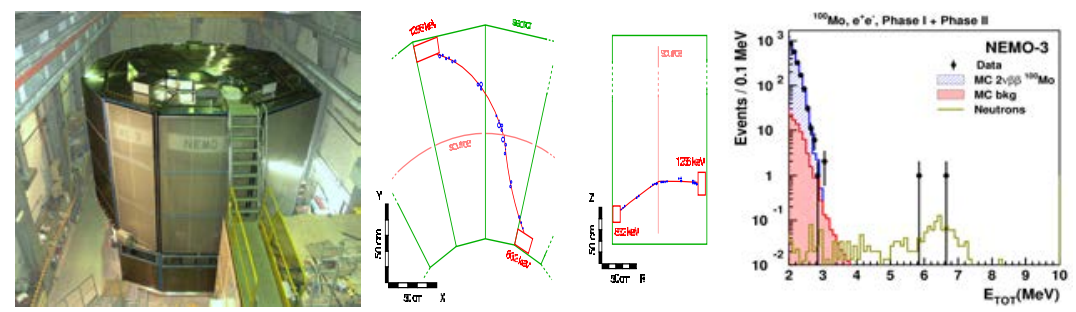

Figure 9: NEMO-3. Left : Photo of the detector at LSM. Middle : Reconstruction of a 2-electron event. Right : 2-electron energy spectrum for ${ }^{100} \mathrm{Mo}$

No events were observed in the [3.2-10] MeV energy range for a $47 \mathrm{~kg} . \mathrm{y}$ exposure. This is very promising for high- $\mathrm{Q}_{\beta}$ isotopes $\left({ }^{48} \mathrm{Ca},{ }^{150} \mathrm{Nd},{ }^{96} \mathrm{Zr}\right)$. Also limits were sets on right handed currents and supersymmetry.

\section{SuperNEMO}

\begin{tabular}{|c|c|c|}
\hline & NEMO-3 & SuperNEMO \\
\hline Isotope & ${ }^{100} \mathrm{Mo}$ & ${ }^{82} \mathrm{Se}\left({ }^{150} \mathrm{Nd},{ }^{48} \mathrm{Ca}\right)$ \\
Calorimeter energy resolution FWHM at $1 \mathrm{MeV}$ & {$[14-17] \%$} & $8 \%$ \\
Source radiopurity : ${ }^{208} \mathrm{Tl}(\mu \mathrm{Bq} / \mathrm{kg})$ & $\simeq 100$ & 2 \\
Source radiopurity : ${ }^{214} \mathrm{Bi}(\mu \mathrm{Bq} / \mathrm{kg})$ & $\simeq[60-300]$ & 10 \\
Tracker gas radiopurity : ${ }^{222} \mathrm{Rn}\left(\mathrm{mBq} / \mathrm{m}^{3}\right)$ & $\simeq 5$ & 0.15 \\
\hline
\end{tabular}

The SuperNEMO project should host $\simeq 100 \mathrm{~kg}$ of $\beta \beta$ isotopes. After a $R \& D$ phase the energy resolution was improved by a factor 2 w.r.t. NEMO-3. An automated drift cells production through a wiring robot was developed.A dedicated detector BiPo-3 to measure the radiopurity of $1.4 \mathrm{~kg}$ of source at the 
required sensitivity level was constructed and installed in Canfranc (see figure 2.5). Against Radon background, emanation measurements were done and the gas purification, permeability of materials were studied.
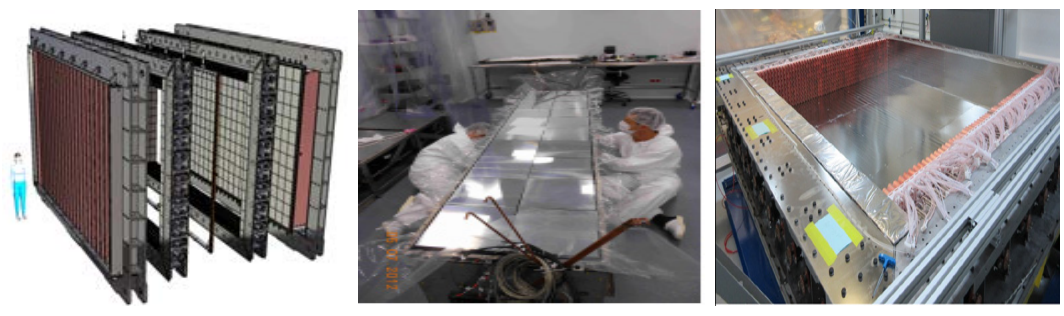

Figure 10: SuperNEMO. Left : Scheme of the tracker, calorimeter of one SuperNEMO module. Middle : BiPo-3 detector at Canfranc. Right : Part of the SuperNEMO demonstrator tracker, during assembly at MSSL.

The first module of SuperNEMO, called SuperNEMO demonstrator, will host $7 \mathrm{~kg}$ of ${ }^{82} \mathrm{Se}$ in the LSM. It should demonstrate the ability to reach SuperNEMO specifications for background. The expected sensitivity is : $\mathrm{T}_{1 / 2}^{0 \nu}>6.510^{24} \mathrm{y}$ $(90 \% \mathrm{CL}),\left|\mathrm{m}_{\beta \beta}\right|<[0.2-0.4] \mathrm{eV}$ with a $17.5 \mathrm{~kg} . \mathrm{y}$ exposure. The detector is in construction and commissioning expected in 2016.

\section{Conclusion}

This review is not exhaustive. The recommandation of the NUSAG american committee is to wait for the results of the demonstrators to choose the best technique(s).

\section{References}

[1] M. Agostini et al., Phys. Rev. Lett. 111, 122503 (2013).

[2] K. Alfonso et al., Phys. Rev. Lett. 115, 102502 (2015),

[3] J. B. Albert et al., Nature 510, 229 (2014),

[4] A. Gando et al., Phys. Rev. Lett. 110, 062502 (2013),

[5] R. Arnold et al,, Phys. Rev. D 92, 072011 (2015). 